\title{
The Economic Vector Space and the Economic Cycle
}

Paul T E Cusack*

1641 Sandy Point Rd, Saint John, NB, Canada E2K 5E8, Canada

*Corresponding author: Cusack PTE, Independent Researcher, BSc E, DULE, 1641 Sandy Point Rd, Saint John, NB, Canada E2K 5E8, Canada, Tel: (506) 214-3313; E-mail: St-michael@hotmail.com

Received date: January 17, 2017, Accepted date: March 22, 2017, Published date: March 29, 2017

Copyright: (c) 2017 Cusack PTE. This is an open-access article distributed under the terms of the Creative Commons Attribution License, which permits unrestricted use, distribution, and reproduction in any medium, provided the original author and source are credited.

\section{Abstract}

A vector space is a series of vectors of different magnitudes and direction which are connected head to tail. There is a vector space that applies to the US economy, for example. My previous paper on the Cusack US Economy Equation contains the 11 variables with the 12th one being $t$

Keywords: Vector space; Macroeconomics; Physical economics

\section{Introduction}

I illustrate here the vector space for the economy as opposed to the physical universe. I've written yet another paper on Cusack's Physical Economics (Figure 1) [1,2].

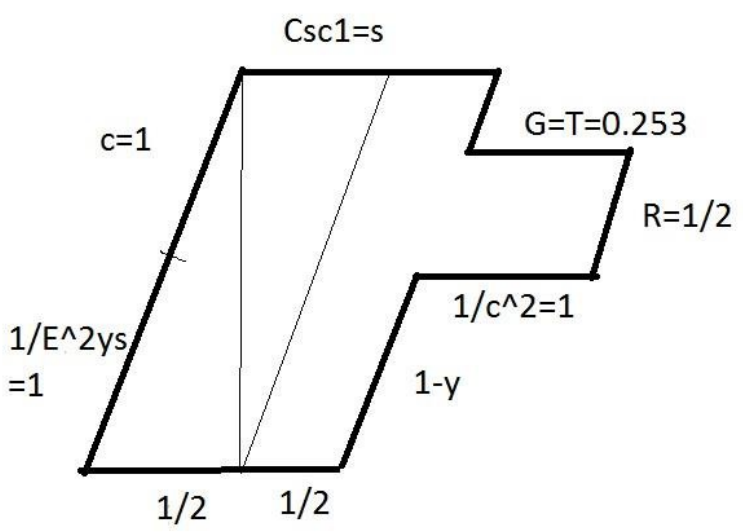

Figure 1: Illustrate 1 on Cusack's Physical Economics.

\section{$1 / 81=0.012345679 \quad 81=\mathrm{c}^{4}=$ speed of light ${ }^{4}$}

Each successive digit is adding 1 less power of 10. So, from logic, a binomial tree yields:

$$
\begin{aligned}
& (1+\mathrm{t})^{11}=\mathrm{e}^{0}+\mathrm{e}^{1}+\mathrm{e}^{1} \ldots \\
& =\left(1 / 7+7 \mathrm{e}^{\mathrm{t}}\right) \\
& =1 / 81 \\
& 0.01234567-1 / 7=7 \mathrm{e}^{\mathrm{t}} \\
& \mathrm{t}=398 \sim 396
\end{aligned}
$$

The plot above is $396 \times 396$

$396+396+396+396=4(386)=0.1584=1-\sin 1=1-\cos 1$

\section{$1-1584=0.8416$}

This is the ideal balance for maximum sustainable output $\mathrm{Y}$.

Sin and cos are a two pole problem since $\mathrm{Y}=1$ at full output and $\mathrm{Y}=0$ at no output (Figure 2).

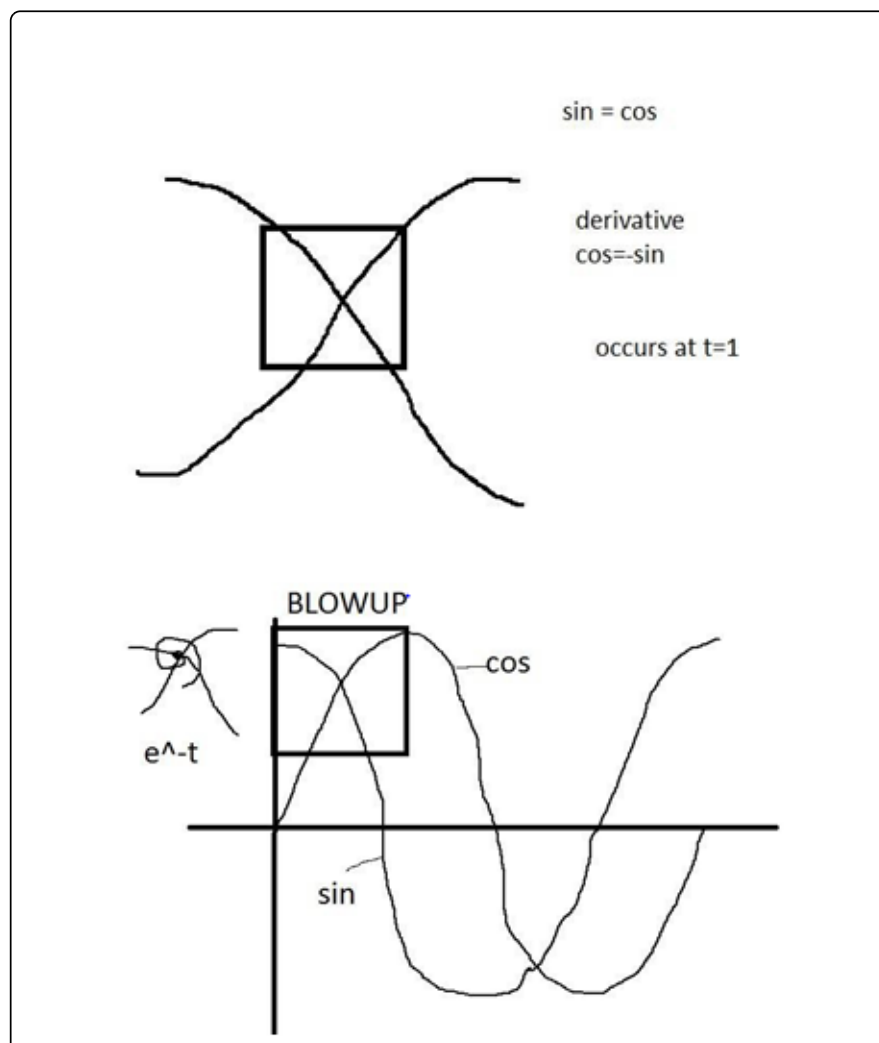

Figure 2: Balance for maximum sustainable.

Now,

The cross product is:

$\| \mathrm{E}|||| \mathrm{t}|| \cos 60=\{\mathrm{G}, \mathrm{ec}\}$ 
$\mathrm{E}=\mathrm{t}$

$\mathrm{E}^{2} \times 1 / 2=\mathrm{G}=0$.

So there is a gravitational equivalent in the economy (Figure 3).

Why is it?

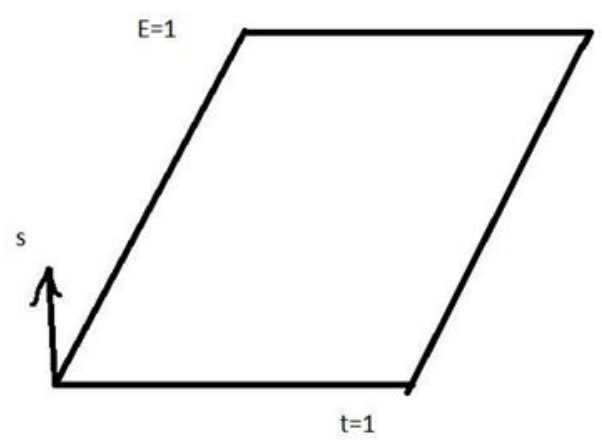

Figure 3: Gravitational equivalent in the economy.

From Newton, we know:

$\mathrm{F}=\mathrm{GM} 1 \mathrm{M} 2 / \mathrm{R}^{2}$

$2.667=8 / 2=2|\mathrm{D}| / \mathrm{c}=\{\mathrm{Gec}\}$ (Infinity) $/ \mathrm{R}^{2}$

$\mathrm{Gec}=0.251 \sim 0.253=$ Period $\mathrm{T}$ (seconds)

$\mathrm{R}=0.3068$

$1 / \mathrm{R}=3.25=13 / 4$

The economy is like a pendulum ranging between high and low outputs.

$$
\begin{aligned}
& x=1 /[x-1] \\
& x^{2}-x-1=0 \\
& x=1.618=\text { Golden mean }
\end{aligned}
$$

Eigen value $=\mathrm{c}=3$

Eigenvector $=\sqrt{ } 3=1.73$

Eigen vector ${ }^{2}=$ eigenvalue

Continuing,

$\mathrm{y}=\mathrm{y}^{\prime}$ is telescopic

$\mathrm{y}=\mathrm{e}^{\mathrm{x}}=\mathrm{y}^{\prime}=\mathrm{y}^{\prime \prime} \ldots$

$\mathrm{y}=1 /+7 \mathrm{e}^{\mathrm{t}}$

$\mathrm{y}^{\prime}=7 \mathrm{e}^{\mathrm{t}}=1 / \mathrm{c}^{2}=0.111$

$\mathrm{e}^{\mathrm{t}}=0.111 / 7=0.1586$

$\sin 1=\cos 1$

This is where sin meets cos or the settling point between $\mathrm{Y}=0$ and $\mathrm{Y}=1$ (NO output, Full output)

So,

$\mathrm{F}=\mathrm{Gec} \times \mathrm{M}_{1} \mathrm{M}_{2} / \mathrm{R}^{2}$
$0.253 \times($ Infinity $)(0) / 13 / 4)^{2}$

$0.86=8 / 3=2|\mathrm{D}| / \mathrm{c}=\mathrm{E} \times|\mathrm{D}| /$ eigenvalue $=0.778 \mathrm{x}$

$\mathrm{x}=0.1111=1 / \mathrm{c}^{2}=1 /$ eigenvalue $^{2}$

from above

$y^{\prime}=1 / 7+7 \mathrm{e}^{\mathrm{t}}$

$0.86=\mathrm{Y}$ Max sustainable

QED

If you didn't understand that, try this:

Assumption: Money is stored energy. Energy cannot be created nor destroyed. Money cannot be created nor destroyed. Governments cannot create money. The simply devalue their currency by printing money.

From the vector space, we know the economy has a resistance $r$ which is the costs of producing output.

when:

$\mathrm{R}=\mathrm{dR} / \mathrm{dt} \mathrm{NPV}=0 \mathrm{Y}=\mathrm{y}^{\prime} \mathrm{R}=\mathrm{r}^{\prime}$ integrate $\mathrm{R}^{2} / 2=\mathrm{R}$

$\mathrm{R}=0,1 / 2 \mathrm{dR} / \mathrm{dt}=\pi$

$\mathrm{E} / \mathrm{t} \pi / 2 \pi=1 / 2=\mathrm{R}$

When $\mathrm{E}=\pi$

$A=\pi R^{2} ; A^{\prime}=2 \pi r$

Circle $=2 \pi r$ A' $=$ Circle $R^{2}=A / \pi R=\sqrt{ } A / \sqrt{ } \pi$

$\mathrm{R}=1 / 2=\sqrt{ } \mathrm{A} / 1.7725$

$\mathrm{A}=\pi / 4=45$ degrees which is the minimum energy level.

The economy seeks the minimum energy to produce maximum sustainable possible output.

So $\mathrm{y}=\mathrm{y}^{\prime}$

$\mathrm{E}=1 / \mathrm{t}$

Work $\mathrm{W}=\mathrm{F} \times \mathrm{d} ; \mathrm{d}=\mathrm{s}$

$=\mathrm{J} / \mathrm{s}=\mathrm{E} / \mathrm{s}$

$\mathrm{W}=\mathrm{E} / \mathrm{t}=\mathrm{E}=1 / \mathrm{t}$

$\mathrm{E} / \mathrm{t}=\mathrm{E}^{2}$

$\mathrm{E}=0 ; 1 / \mathrm{t}=\mathrm{Wt}=1 / \mathrm{t}$

$\mathrm{Y}=\mathrm{W} \mathrm{Y}^{\prime}=\{\mathrm{F} \times \mathrm{d}\}^{\prime}$

$\mathrm{Y}^{\prime}=\mathrm{F} \times\left(\mathrm{ds} / \mathrm{dt} \mathrm{Y}^{\prime}=\mathrm{Fv}=(\mathrm{Ma})(\mathrm{P} / \mathrm{v})\right.$

$\mathrm{P}=\mathrm{Mv}$

$\mathrm{Y}^{\prime}=\mathrm{MP} ; \mathrm{Y}^{\prime}=\mathrm{M}(\mathrm{Mv})$

$M^{2}=1 M=\sqrt{ } 1=-1 M=1$

Now, $\mathrm{Wt}^{2}=1 \mathrm{~W}=1$

$\mathrm{W}=\mathrm{J} / \mathrm{s} ; \mathrm{W}=\mathrm{y} / 1$

$\mathrm{W}=\mathrm{y}=1 ; \mathrm{W}=1 ; \mathrm{y}=1 ; \mathrm{M}=1$

$\mathrm{t}=1$

$y=F ; y^{\prime}=-F$ 
$\mathrm{y}=\mathrm{W}$ and $\mathrm{y}^{\prime}=\mathrm{W}$

Recall Money is stored work. So Economics follows the same laws as the universe.

$\mathrm{Y}^{\prime}=\mathrm{F}(\mathrm{ds} / \mathrm{dt}) \mathrm{Y}^{\prime}=(1)(\mathrm{ds} / \mathrm{dt})$

$\mathrm{Y}^{\prime}=\mathrm{ds} / \mathrm{dt}$

$\mathrm{Y}=$ Integral ds $/ \mathrm{dt} \mathrm{Y}^{2} / 2=\mathrm{s}+\mathrm{C}_{1} \mathrm{Y}=\sqrt{2}+\mathrm{C}_{1}$

$\mathrm{Y}=\sin 1$

$\mathrm{Y}^{\prime}=-\cos 1$

$\mathrm{Y}=-\mathrm{y}^{\prime}=\sin 1=\cos 1$

Vector spaces components:

Period: $\mathrm{T}=1 / \mathrm{t}=\mathrm{E}=\mathrm{W} \times \mathrm{t} ; 1 / \mathrm{t}=\mathrm{Wt}$

$\mathrm{T}^{2} \mathrm{~W}=1 ; \mathrm{T}(\mathrm{F} \times \mathrm{d})=1$

$\mathrm{T}^{2} \times \mathrm{y} \times \mathrm{s}=1$

(1) $(\mathrm{y}(1))=1 \mathrm{Y}=1$

Speed of light: $C^{2}-c-1=2 c-1 C=1$

Force

$\mathrm{Y}=\mathrm{F}$

$\mathrm{W}=\mathrm{F} \times \mathrm{d}$

$1=(\sin 1)(d)$

$=1 / \sin 1=\mathrm{d}=\mathrm{s}$

$\csc 1=s$

$\mathrm{s}=1.11884$

$1-\sin 1=0$

$-\sin =-F ; 0.84=F ; F=y$

$\mathrm{Y}=\sin 1$

$(\pi-\mathrm{e})=\mathrm{m}=\mathrm{E} / \mathrm{cuz}$

$\mathrm{Y}=\mathrm{mx}+\mathrm{b} ; \mathrm{Y}=0.4233 \mathrm{x}+0$

$\mathrm{Y}=\operatorname{cuz} \times \mathrm{x}$

$Y=R \times x ; A=\pi \times R^{2} ; A^{\prime}=2 \pi \times R ; C=2 \pi R ; A^{\prime}=C$

$2 \pi \mathrm{R}=2 \pi \mathrm{u} ; \mathrm{R}=\mathrm{rx} ; \mathrm{X}=2 \pi ; \mathrm{T}=2 \pi$;

The economic cycle:

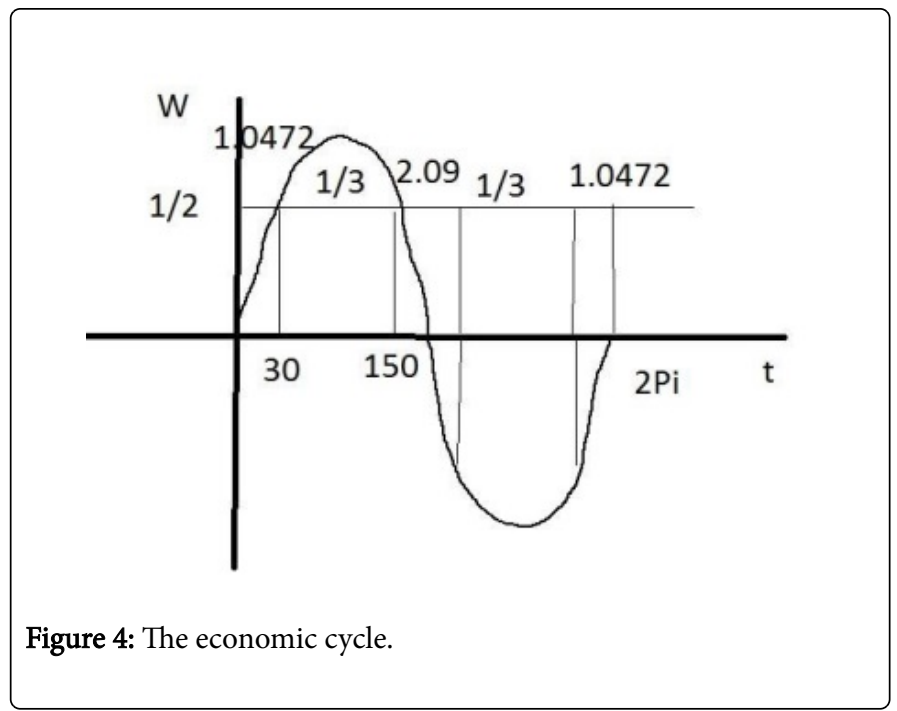

Boom $=2.09$ years stagnation $=4.18$ years decline $=2.09$ years $=1$ economic cycle $=8.36$ years (Figures 4 and 5 ).

So, we had a decline starting in 2007.9. Add a cycle we get 2014.17=March 2014 .

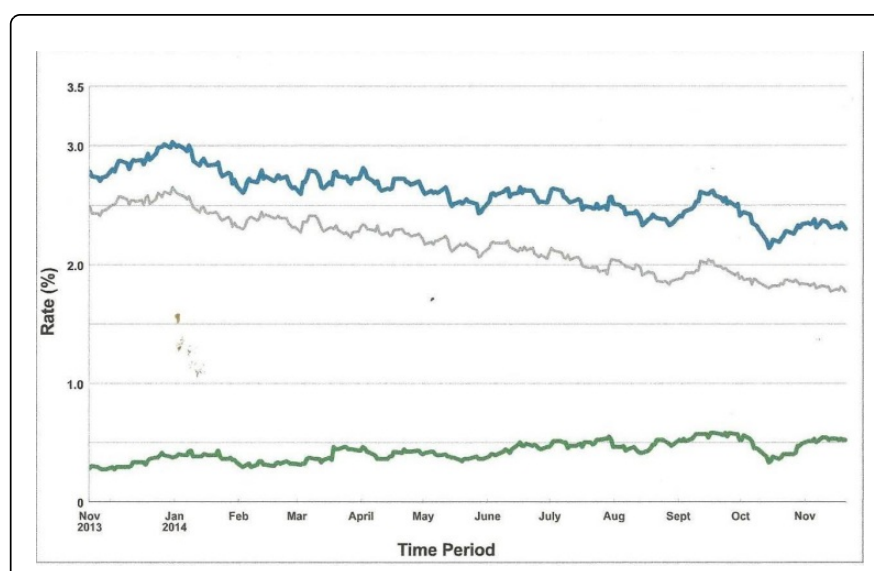

Figure 5: Graph for economic cycle.

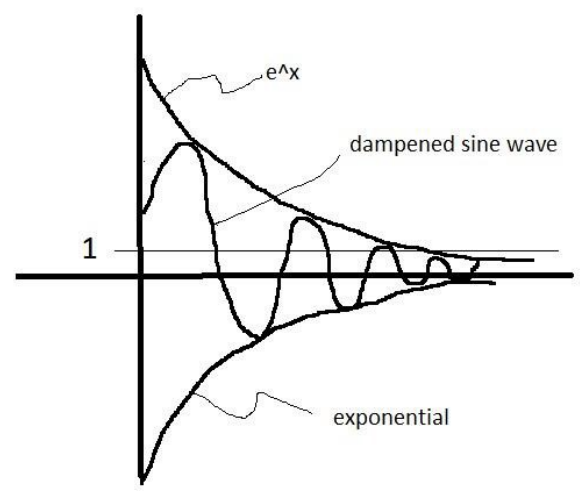

Figure 6: Cycles from depression to depression. 
Page 4 of 4

Profits go toward zero. So e- $\mathrm{x}=0 \mathrm{x}=\operatorname{Ln} 0 \mathrm{x}=\mathrm{t}=1 \mathrm{Y}=\mathrm{e} 1 \cos [2 \pi(1)$
$\mathrm{Y}=2.71828$
$\mathrm{y}=\mathrm{y}^{\prime}$
And,
9.44 cycles from depression to depression $\mathrm{Y}=\mathrm{e}^{-9.44} \times \cos (2 \pi \times 9.44)$
$\mathrm{Y}=7.5$
$1 / \mathrm{Y}=0.1334=\mathrm{s}$
Circle $=\mathrm{s}^{2}$
Circle $=0.1334^{2}$ Circle $=0.0178$

Profits go toward zero. So $\mathrm{e}-\mathrm{x}=0 \mathrm{x}=\mathrm{Ln} 0 \mathrm{x}=\mathrm{t}=1 \quad \mathrm{Y}=\mathrm{e} 1 \cos [2 \pi(1)]$ $=2.71828$

Circle $=0.1334^{2}$ Circle $=0.0178$
$\mathrm{C}=2 \pi \mathrm{r}$ Circle $=2.8323$

\section{Conclusion}

The mathematics models of physics can be used to solve outstanding economic problems.

\section{References}

1. Cusack P (2010) How Politics Killed a Boom Town. Saint John, New Brunswick.

2. Cusack P (2010) Engineered Solutions to Economic Problems. 